\title{
Gardasil - preventing cervical cancer and other diseases related to HPV
}

\author{
Scott Chambers
}

CSF Medical Communications, Cheltenham, UK

Drugs in Context

DOI: http://dx.doi.org/10.7573/dic.212216

Citation: Chambers, S. Gardasil - preventing cervical cancer and other diseases related to HPV. Drugs in Context: e212216. doi:10.7573/dic.212216

Copyright: this is an open access article published under the terms of the Creative Commons License Deed (CG BY-NC-ND 3.0) which allows you to share, copy, distribute and transmit the work provided it is properly attributed. You may not use this work for commercial purposes. For further information on commercial use, contact publisher@justmedicalmedia.com or go to wrwre.drugsincontext.com/copyright.

Search criteria: English language articles were identified by searching the PubMed database using the search terms 'Gardasil' and 'HPV vaccine'. Abstracts were evaluated and selected for further review according to our standard protocols. Bibliographies of individual articles were also assessed for additional articles of interest and the manufacturer of Gardasil was contacted and was invited to supply any additional data to that identified via the PubMed database.

Date of last literature search: 19 November 2007.

Conclusion: Gardasil offers a high level of protection against high-grade cervical lesions and adenocarcinoma in situ in HPV-naïve women. The impact of Gardasil on the incidence of cervical cancer will depend on the wide-scale implementation of appropriate immunisation programmes, though cervical screening should continue for the foreseeable future to provide maximum benefit.

NB: This article was originally published by CSF Medical Communications Ltd (CSF) in Drugs in Context 2008;4(1):29-40. Drugs in Context and all CSF copyrights were acquired by Just Medical Media Ltd in 2009. 


\section{SUMMARY}

Human papillomavirus (HPV) is absolutely required for the development and progression of precancerous lesions in the cervical epithelium, and has been isolated in more than $99 \%$ of cervical cancers sampled. Given that the majority $(\sim 70 \%)$ of cases of cervical cancer are caused by only two HPV types, appropriately designed vaccines are an attractive proposition for preventing HPV infection and thus, by extension, cervical cancer. Gardasil is a recombinant quadrivalent HPV vaccine comprising a mixture of highly purified virus-like particles (VLPs) of the major capsid protein (L1) derived from HPV types 6, 11, 16 and 18. Gardasil prevents persistent infection by the vaccine HPV types for at least 5 years. More pertinently, in HPV-naive women, Gardasil provides complete protection against high-grade cervical dysplasia. The vaccine also prevents vulval precancers, reduces the incidence of vaginal precancerous lesions and protects against genital warts caused by the vaccine HPV types. The vaccine stimulates a robust immune response, with serum anti-HPV antibody titres substantially higher than those associated with a natural HPV infection, whilst immune responses in children are non-inferior to those of older adolescents and young women, suggesting that Gardasil also prevents HPV infection in this population. Gardasil is well tolerated with a favourable safety profile. In summary, the introduction of Gardasil in conjunction with cervical screening has the potential to substantially reduce the burden of cervical cancer and other HPV-related diseases.

Key words: human papillomavirus; HPV; cervical intraepithelial neoplasia; cervical cancer; genital warts; Gardasi ${ }^{\circledR}$.

\section{HPV AND CERVICAL CANCER: A PERSPECTIVE}

Worldwide, cervical cancer is the most common female malignancy after breast cancer. ${ }^{1}$ In 2002, more than 493,000 new cases of invasive cervical cancer were diagnosed with about 274,000 deaths reported, representing about $10 \%$ of all female cancer deaths. ${ }^{2}$ The majority of these cases $(\sim 80 \%)$ occurred in the developing world, ${ }^{3,4}$ primarily because of difficulties in implementing population-wide cervical screening programmes. ${ }^{4}$ In many countries of the developing world, cervical cancer remains the most common female cancer and is responsible for the loss of an estimated 2.4 million years of life. ${ }^{2}$ In developed countries, mortality rates from invasive cervical cancer have fallen substantially, reflecting the success of cervical screening programmes in these countries.

HPV is the obligate aetiological agent necessary for the development of cervical cancer, with the virus detected in more than $99 \%$ of cervical cancers sampled. ${ }^{5}$ In addition, HPV plays an important pathophysiological role in the development of other anogenital cancers, including cancers of the vulva, vagina, penis and anus. ${ }^{6}$ Certain low-risk HPV types are also responsible for the development of anogenital warts, which are extremely prevalent in the population, are highly distressing for the patient and are difficult and costly to manage from a healthcare professional perspective. ${ }^{7}$ Preventing persistent HPV infection through prophylactic vaccination offers a means of substantially reducing the huge clinical and socioeconomic burden of these devastating diseases. Of the 100 HPV types identified to date, HPV 6, 11, 16 and 18 appear to cause the majority of HPVrelated anogenital disease. Therefore, the use of a prophylactic vaccine which protects against infection by these virus types can be expected to have the greatest impact on HPV-related disease and its associated socioeconomic burden. ${ }^{1}$ Gardasil is a recombinant quadrivalent HPV vaccine comprising a mixture of highly purified virus-like particles (VLPs - see below) derived from HPV types 6, 11, 16 and 18, the HPV types responsible for the majority of HPV-related morbidity. These VLPs are non-oncogenic, noninfectious and have no disease-causing potential. ${ }^{8}$ 


\section{VACCINE COMPOSITION AND DOSING SCHEDULE}

Gardasil is supplied in a single-dose pre-filled syringe containing $0.5 \mathrm{~mL}$ of white cloudy suspension (after agitation). Each $0.5 \mathrm{~mL}$ dose contains VLPs of the major capsid protein (L1) of HPV types $6(20 \mu \mathrm{g}), 11(40 \mu \mathrm{g}) 16(40 \mu \mathrm{g})$ and $18(20 \mu \mathrm{g})$. The highly purified VLPs are adsorbed on to aluminium hydroxyphosphate sulphate $(225 \mu \mathrm{g}){ }^{9} \quad$ The recommended dosing schedule comprises three separate $0.5 \mathrm{~mL}$ doses with the second and third doses administered 2 months and 6 months after the first dose. Where an alternative schedule is required, the second dose of the vaccine should be administered at least 1 month after the first dose with the third dose given at least 3 months after the second dose. All three doses should be administered within 1 year. Gardasil should be administered intramuscularly, preferably into the deltoid area of the upper arm or into the upper anterolateral region of the thigh. ${ }^{9}$ The need for a booster dose later in life has not yet been established. Although evidence for the duration of protection will not be available for many years, there is preliminary evidence of efficacy and a sustained immune response to the vaccine for up to 5 years. ${ }^{10}$ Gardasil has been administered at the same time as the recombinant hepatitis $\mathrm{B}$ vaccine without altering the immune response to the vaccine HPV types or adversely affecting seroprotection afforded by the hepatitis $\mathrm{B}$ vaccine. ${ }^{8}$ However, the interaction of Gardasil with other vaccines has not yet been studied.

Vaccination against HPV infection should not be regarded as a substitute for routine cervical screening. Gardasil will not protect against infections caused by non-vaccine HPV subtypes or against infections that may have occurred prior to vaccination. Therefore, routine cervical screening remains essential in order to maximise the protection of women against HPV-mediated disease. ${ }^{9}$

\section{KEY CLINICAL DATA \\ The Gardasil clinical trial programme and endpoint selection}

The Gardasil clinical trial programme included two 'proof-of-concept' Phase II trials and two pivotal Phase III trials (termed Females United To Unilaterally Reduce Endo/ectocervical Disease or FUTURE I and II). ${ }^{10-14}$ All studies were randomised and double-blinded, and included a placebo comparator arm. The vaccination schedule in each case was a three-dose course given at day 0 and months 2 and 6. Clinical efficacy was evaluated from month 7 in the perprotocol (PP) population with varying lengths of follow-up thereafter. Study evaluations included gynaecological examinations, cervicovaginal sampling for HPV DNA, serology and Pap testing, and colposcopy. The similarity in study designs permitted the pooling of data in a prespecified integrated analysis. ${ }^{9}$

In total, 20,541 women aged 16-26 years were recruited. No HPV screening phase was performed and thus women were randomised to treatment regardless of their HPV serology or HPV DNA status at baseline. Nevertheless, as Gardasil is a prophylactic vaccine, primary efficacy evaluations were conducted in the cohort who received all three doses of the vaccine within 1 year according to the study schedule, had no major protocol violations and were naïve to vaccine-type HPV for up to 1 month after the final dose. Overall, 73\% of women were naïve to all four HPV vaccine types at enrolment. ${ }^{9}$ Secondary efficacy evaluations took place in a modified intention-to-treat (MITT) population, a group who were negative to the respective HPV type at day 1 or who did not receive the full threedose vaccine schedule. Unless otherwise stated, the majority of data presented in this review pertain to the PP population.

Protection against clinical disease is the most relevant clinical endpoint to establish vaccine efficacy. However, it is unethical and impractical to use cervical cancer as an endpoint in clinical trials. ${ }^{15}$ Therefore, clinically relevant surrogate endpoints that reflect a stage in the 
natural history of clinical disease were used to monitor vaccine efficacy. ${ }^{15}$ As a first step, monitoring persistent HPV infection acts as a useful surrogate marker for disease prevention. After discussions with regulatory authorities, the incidence of CIN2/3 or adenocarcinoma in situ (AIS) was considered to be the most direct correlate of vaccine efficacy in preventing cervical cancer. ${ }^{15}$ The Gardasil clinical trial programme also evaluated vaccine efficacy against low-grade cervical lesions (CIN1), external genital precancerous lesions (VIN and VaIN) and genital warts caused by the vaccine HPV types. Immunogenicity was also evaluated and safety and tolerability recorded.

\section{Immunogenicity}

Based on animal data, Gardasil appears to protect against persistent HPV infection by inducing typespecific neutralising antibodies through a humoral response to the L1 capsid antigens of the four HPV vaccine types. ${ }^{20}$ However, a serological correlate of immunity has not yet been identified in humans and thus the minimum antibody titre required to confer protection against HPV infection is not yet known. ${ }^{1}$ A series of studies in the Gardasil clinical trial programme employed competitive immunoassays to evaluate the immune response to vaccination. ${ }^{15}$ Peak antibody titres against all four HPV vaccine types were reported in more than $99.5 \%$ of patients, 1 month after completion of the three-dose vaccine schedule, indicating a robust immune response (Figure 1). ${ }^{12}$ Titres then declined until month 18 when the rate of decline slowed and then stabilised (Figure 1). ${ }^{12}$ Gardasil also induces a more robust immune response than does a natural infection (Figure 1). ${ }^{12}$ Longerterm studies have demonstrated that the immune response persists for at least 5 years, with mean antibody titres consistently remaining above those

Figure 1. Anti-HPV geometric mean titres (GMT) for Gardasil and placebo recipients according to HPV status at recruitment. ${ }^{12}$
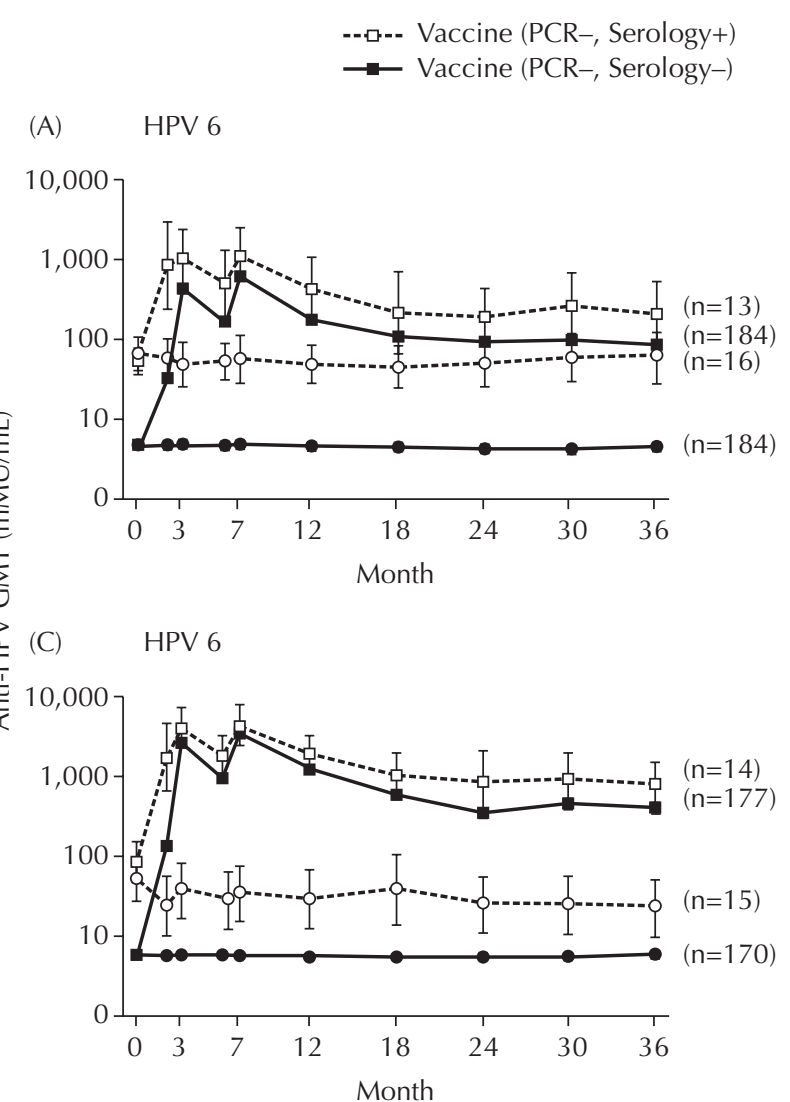

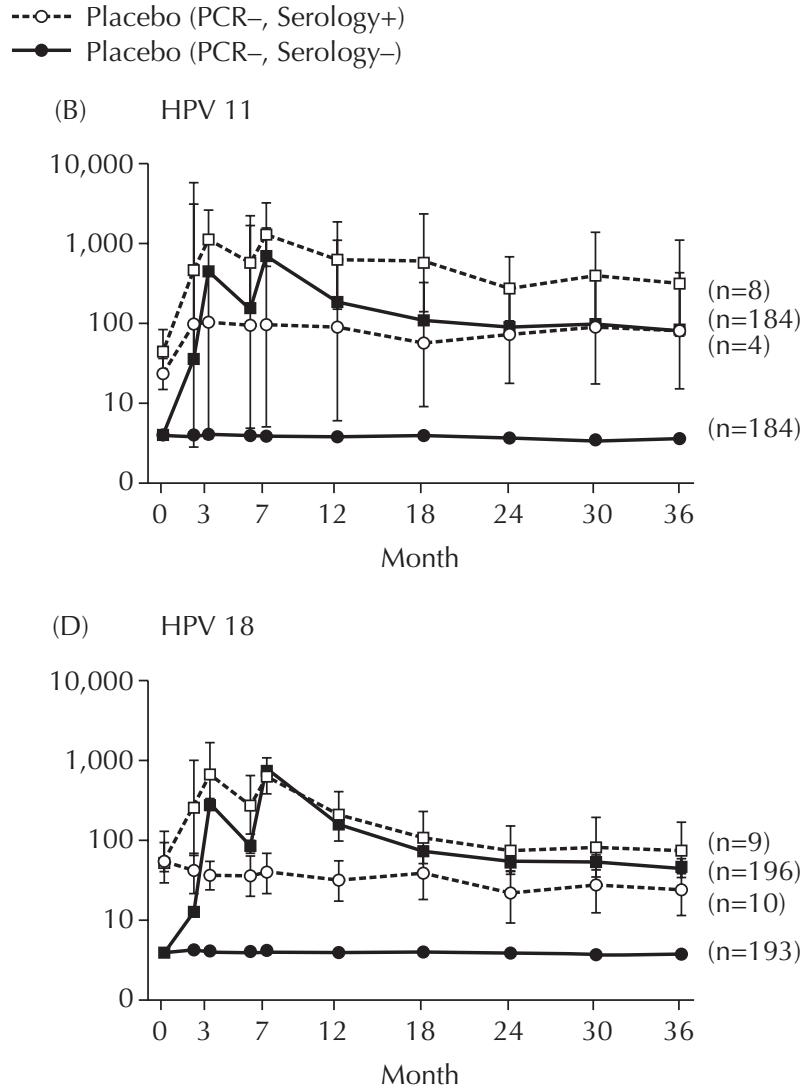

Drugs in Context e212216 | www.drugsincontext.com 
associated with a natural infection. ${ }^{10}$ Gardasil also stimulates an anamnestic response in women with evidence of prior natural HPV infection (i.e. those who were seropositive for one of the vaccine types at enrolment but who subsequently cleared the infection). ${ }^{12}$ These women have an immune response to the vaccine which is approximately double in magnitude to HPV-naïve women, suggestive of the establishment of B-cell memory. ${ }^{1}$

\section{Efficacy against persistent HPV infection}

Two Phase II studies utilised a virological endpoint of persistent HPV infection as a surrogate for vaccine efficacy. ${ }^{11,16}$ Persistent infection was defined as the presence of vaccine HPV DNA at two or more consecutive visits at least 4 months apart or at the final scheduled follow-up visit. The first study evaluated only the HPV 16 component of the vaccine, ${ }^{16}$ although this was a reasonable evaluation given that HPV 16 is responsible for more than $50 \%$ of all cervical cancers. ${ }^{15}$ Two reports from this study have been published: one an interim analysis presenting data from a median of 17.4 months' follow-up, ${ }^{16}$ the other extending followup to 3.5 years after completion of the vaccine schedule. ${ }^{17}$ At the interim evaluation, 41 cases of persistent HPV 16 infection were reported in the PP population, all in the placebo group, representing a protective efficacy of the vaccine of $100 \%$ (95\% CI: $90-100 ; p<0.001) .{ }^{16}$ Of these, nine were associated with CIN1/2. By the end of the study (i.e. 4 years after randomisation), protective efficacy was reported as 94\% (95\% CI: 88-98), with seven persistent HPV 16 infections occurring in the vaccine group compared with 111 in the placebo group. ${ }^{17}$ The seven vaccine cases had HPV 16 DNA detected at the last study visit without observed persistence. ${ }^{6}$ No cases of CIN were observed in the vaccine group, with a further three cases found in the placebo group (yielding a total of 12 cases over the course of the study), thereby representing a protective efficacy against CIN of $100 \%$ [95\% CI: $65-100]$ ]. ${ }^{17}$

The first evaluation of the quadrivalent HPV vaccine was a two-part dose-escalation and doseranging study. ${ }^{11}$ This study evaluated vaccine efficacy against a slightly different endpoint: the combined incidence of infection with HPV vaccine types or cervical or external genital HPVassociated disease. A total of 277 and 275 women were randomised to low-dose quadrivalent vaccine (equivalent to Gardasil) and placebo, respectively. After 2.5 years' follow-up from completion of the schedule, the primary endpoint had occurred in four of the 235 women in the vaccine group and 36 of the 233 placebo subjects. This represented a protective efficacy of $90 \%(95 \%$ CI: $71-97$; $p<0.0001)$. Three of the four vaccinees testing positive for HPV infection had HPV 16 detected at the final study visit before being lost to followup, with the remaining subject testing positive for HPV 18 at months 12 and 18, which had subsequently cleared by completion of the study. No cases of clinical disease associated with HPV $6 / 11 / 16 / 18$ infection were reported in the vaccine group compared with six cases (three CIN and three genital lesions) in the placebo group.

Five-year follow-up data have also been reported from a subset of the participants in this study $(\mathrm{n}=226)$, representing the longest evaluation of Gardasil's efficacy published to date. ${ }^{10}$ Two primary endpoints occurred during the extension phase in the vaccine group, both of which had already been described in the initial phase. ${ }^{11}$ In contrast, a further 11 new cases of persistent HPV infection occurred in the placebo group, yielding a total of 47 cases after 5 years. This represents a protective efficacy of the vaccine of $95.6 \%$ (95\% CI: 83.3-99.5). No additional cases of CIN or external genital lesions were reported during the extension phase of this analysis, which translates to $100 \%$ efficacy (95\% CI: 12.4-100) of Gardasil against these lesions over 5 years. ${ }^{10}$ This provides encouraging evidence of the protective efficacy of the vaccine against these clinically relevant lesions. 


\section{Efficacy against high-grade cervical lesions}

FUTURE I and II prospectively evaluated the efficacy of Gardasil against high-grade precancerous cervical lesions and clinical disease in a very large population of females aged 16-23 years. ${ }^{13,14}$ FUTURE II remains the largest study that has evaluated the efficacy of Gardasil in preventing high-grade cervical dysplasia, AIS and cervical cancer caused by HPV types 16 and 18. ${ }^{14}$ FUTURE I also assessed vaccine efficacy against high-grade cervical dysplasia (CIN2/3) in addition to low-grade cervical lesions (CIN1) and external genital lesions including VIN, vaginal intraepithelial neoplasia (VaIN) and genital warts caused by HPV types 6, 11, 16 and $18 .^{13}$ Efficacy data from these studies and the pooled datasets from the entire Gardasil clinical trial programme showing vaccine efficacy against high-grade cervical dysplasia (CIN2/3) or worse are summarised in Table 1.

FUTURE II recruited 12,167 women from 90 centres in 13 countries and employed a primary endpoint of HPV 16/18-related CIN2/3, AIS and cervical cancer. ${ }^{14}$ Evaluations, including Pap tests, cervicovaginal sampling and genital examinations, were conducted on an annual basis, which is more reflective of standard clinical practice than the intensive follow-up used in the FUTURE I programme (see below). ${ }^{9}$ Although data from the study are currently unpublished, 2-year interim efficacy data were presented at the Annual Meeting of the Infectious Diseases Society of America (IDSA) in October 2005. ${ }^{14}$ In the PP population, no primary events occurred amongst the 5,301 vaccinated women compared with 21 events in the 5,258 women given placebo. This reflects $100 \%$ protective efficacy $(95 \%$ CI: 80.9-100; $p<0.001$ ) of Gardasil in the prevention of HPV 16- or HPV 18-related CIN2/3 or AIS (Figure 2). In the MITT analysis, one case of CIN2/3 or AIS was observed amongst 5,736 women compared with 36 in the placebo group, corresponding to a protective efficacy of $97 \%$ (95\% CI 83-100; $p<0.001$ [Figure 2]), further supporting the observations made in the PP population.

Two-year data have also been presented from FUTURE I with regard to vaccine efficacy against CIN, AIS and cervical cancer. ${ }^{13}$ FUTURE I enrolled 5,442 adolescents and women and adopted an intensive visit schedule,

Table 1. Efficacy of Gardasil against precancerous cervical lesions (CIN2/3), adenocarcinoma in situ (AIS) or cervical cancer. Data from the per protocol population. ${ }^{9,10,11,13,14,16,17}$

\begin{tabular}{|c|c|c|c|c|c|}
\hline \multirow[t]{3}{*}{ Clinical trial } & \multicolumn{4}{|c|}{ Intervention } & \multirow{3}{*}{$\begin{array}{l}\text { Efficacy } \\
(95 \% \mathrm{Cl})\end{array}$} \\
\hline & \multicolumn{2}{|c|}{ Gardasil } & \multicolumn{2}{|c|}{ Placebo } & \\
\hline & $\begin{array}{l}\text { Evaluable } \\
\text { population }\end{array}$ & $\begin{array}{l}\text { Number } \\
\text { of cases }\end{array}$ & $\begin{array}{l}\text { Evaluable } \\
\text { population }\end{array}$ & $\begin{array}{l}\text { Number } \\
\text { of cases }\end{array}$ & \\
\hline Protocol $005^{16,17}$ & 755 & 0 & 750 & 12 & $\begin{array}{l}100 \% \\
(65-100)\end{array}$ \\
\hline Protocol $007^{10,11}$ & 231 & 0 & 230 & 1 & $\begin{array}{l}100 \% \\
(<0-100)\end{array}$ \\
\hline $\begin{array}{l}\text { Protocol } 0013^{13} \\
\text { (FUTURE I) }\end{array}$ & 2,200 & 0 & 2,222 & 19 & $\begin{array}{l}100 \% \\
(78.5-100)\end{array}$ \\
\hline $\begin{array}{l}\text { Protocol } 0015^{14} \\
\text { (FUTURE II) }\end{array}$ & 5,301 & 0 & 5,258 & 21 & $\begin{array}{l}100 \% \\
(80.9-100)\end{array}$ \\
\hline Combined analysis & 8,487 & $\mathbf{0}$ & 8,460 & 53 & $\begin{array}{l}100 \% \\
(92.9-100)\end{array}$ \\
\hline
\end{tabular}

NB Protocol 005 evaluated only the HPV 16 VLP component of Gardasil. Data presented are for the 4-year follow-up in this study. ${ }^{17}$ 
with evaluations conducted every 6 months. Adopting the same endpoint as FUTURE II (incidence of HPV 16/18-related CIN2/3, AIS and cervical cancer), vaccine efficacy was again $100 \%$ in the PP population (95\% CI: 78.5-100). ${ }^{13}$

Finally, the pooled data analysis also reported 100\% protective efficacy (95\% CI: 92.9-100) against HPV 16/18-related CIN2/3, AIS and cervical cancer (Table 1). ${ }^{9}$ The corresponding protective efficacy in the MITT population in the combined analysis was 99\% (95\% CI 93-100; $p<0.001)$.

\section{Efficacy against low-grade cervical lesions}

Low-grade cervical lesions frequently regress without the need for further intervention. However, if low-grade lesions are detected, further follow-up is indicated, which is costly to the health service and emotionally distressing to the patient. Therefore, any intervention that has an impact on low-grade lesions could be expected to reduce this clinical and socioeconomic burden. FUTURE II demonstrated

Figure 2. Efficacy of Gardasil against precancerous cervical lesions (CIN2/3), adenocarcinoma in situ (AIS) or cervical cancer. Data from the per protocol (PP) and the modified intention-to-treat (MITT) populations in the FUTURE II trial. ${ }^{14}$

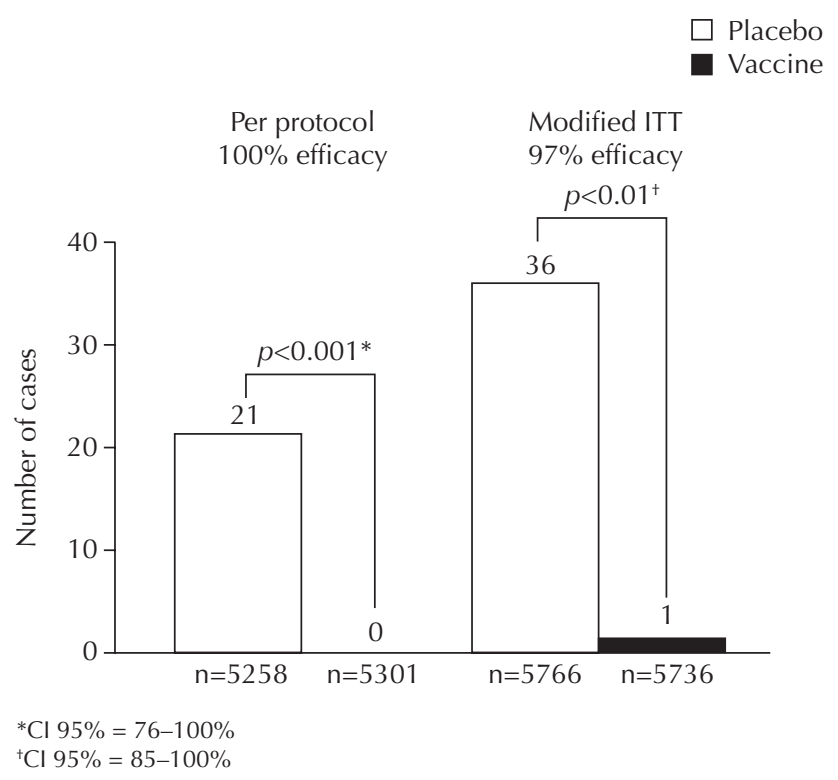

that Gardasil had 100\% efficacy (95\% CI: 84.1100) against low-grade pre-cancerous cervical abnormalities due to HPV 6, 11, 16 and $18 .{ }^{13}$ By combining relevant data from the appropriate studies which evaluated low-grade lesions as a clinical endpoint, the efficacy of Gardasil was reported to be $93.1 \%$ (95\% CI: 81.4-98.2).

\section{Efficacy against external genital lesions}

Genital warts exact a substantial burden of morbidity and cause significant emotional distress to affected individuals. ${ }^{1,18}$ Genital warts are also costly and difficult to treat, patients frequently fail to respond to treatment and warts often recur. Therefore, any intervention that reduces the burden of these lesions should be welcomed. In FUTURE I, Gardasil had 100\% efficacy (95\% CI: 86.4-100) at preventing genital warts caused by HPV 6, 11, 16 and $18 .{ }^{13}$ In the integrated analysis, one additional case of genital warts was reported (reported in FUTURE II), thereby reducing the overall protective efficacy of the vaccine against these lesions to $98.9 \%$ (95\% CI: 93.7-100).

Persistent HPV infections are also related to the development of precancerous vulval and vaginal lesions. Data from the combined analysis, reveal that Gardasil confers 100\% protective efficacy against VIN2/3 (95\% CI: 41.4-100). ${ }^{9}$ Gardasil also appears to reduce the incidence of VaIN $2 / 3$, although due to the limited number of vaginal lesions observed in these trials, statistical significance was not achieved in this endpoint. In total, eight cases of VIN2/3 and five cases of VaIN2/3 were recorded in the combined analysis, all of which occurred in the placebo group.

\section{Efficacy in women with prior HPV infection}

The design of the studies in the Gardasil clinical trial programme allowed for an evaluation of vaccine efficacy against the remaining HPV subtypes in a group of women who were DNA- or antibodypositive to one or more vaccine HPV type. Pooled 
data from the MITT population were evaluated in order to determine whether Gardasil protected against clinical disease caused by the HPV types to which these subjects remained naïve. ${ }^{19}$ Overall, $27 \%$ of the population had evidence of prior infection with a vaccine HPV type. Of these $74 \%$ were positive to only one HPV type and had no evidence of infection by the remaining virus types. ${ }^{2}$ After an average of 24 months' followup, Gardasil remained 100\% effective (95\% CI: 55-100) at preventing CIN 2/3, cervical cancer or AIS caused by the remaining HPV types. The vaccine was also highly effective against external genital warts, VIN1-3 and VaIN1-3 in this group of women.

\section{Efficacy in children}

Evaluating vaccine efficacy in younger children is complicated by the fact that there is a low rate of HPV exposure in this sexually naïve population. In addition, ethical and legal constraints pertaining to the evaluation of sexual activity in such a young population make evaluation of clinical protection problematic. Therefore, in an effort to determine the effectiveness of Gardasil in a younger cohort of children, immunogenicity was determined in a group of sexually naïe boys and girls aged 10-15 years $(n=1,016) .{ }^{22}$ Immune responses from these groups were compared with those of a group of older adolescents and young women aged 16-23 years $(n=513)$, a population for whom vaccine efficacy had already been demonstrated. If the immune response to the vaccine in the younger age group was non-inferior to that of the older cohort then the efficacy data could effectively be 'bridged' to the younger age group.

As with the other Gardasil studies, the three cohorts were randomised to a three-dose vaccination schedule at day 1 , month 2 and month 6. Serum samples were taken at each immunisation visit, with an immunoassay performed to determine the immune response at day 1 , month 3 and month 7. To meet the criterion for non-inferiority between the study cohorts, the lower boundary of the $95 \%$ confidence interval for the ratio of geometric mean titres (GMTs) between the older and younger groups was required to exceed 0.5 for all HPV types evaluated. In addition, betweengroup differences in seroconversion rates could not exceed $5 \%$.

By month 7 more than $99.5 \%$ of the study population had seroconverted for the four HPV vaccine types, thus meeting one of the criteria for non-inferiority between the younger and older age cohorts. Mean serum GMTs at month 7 were approximately two-to-three-fold higher in the younger age group compared with older adolescents and young women. In each case, the lower boundary of the $95 \%$ confidence interval exceeded 0.5 for each of the HPV types, thereby satisfying the second criterion for non-inferiority. Additional post hoc analyses demonstrated that individuals who were HPV seropositive on day 1 mounted immune responses to the vaccine that were numerically higher than HPV-naive subjects, indicative of an anamnestic response to the vaccine as reported elsewhere. A further noteworthy observation from this study was the higher (1.07-1.33-fold) serum GMTs reported in boys compared with girls. This non-inferior response in boys provides support for the implementation of gender-neutral HPV vaccination programmes.

In summary, Gardasil stimulates a robust immune response in younger children aged 10-15 years, which is statistically non-inferior to an older cohort of adolescents and women. This observation supports the bridging of efficacy data reported from older populations to younger children. Data from this study formed the basis for the licensing of the vaccine in children from 9-15 years of age.

\section{SAFETY}

More than 20,000 subjects were enrolled in the Gardasil clinical trial programme for whom safety evaluations are available. ${ }^{8}$ Gardasil had a favourable safety profile and was generally well 
tolerated with up to 5 years' follow-up. ${ }^{9}$ The most common adverse events reported were injectionsite reactions and mild fever. Vaccine-related adverse events that occurred with a frequency of at least $1.0 \%$ and at a greater frequency than with placebo are summarised in Table 2. ${ }^{9}$ The majority of these adverse events were rated as mild or moderate in severity with no serious vaccine-related adverse events reported in the clinical trial programme. ${ }^{21}$

Discontinuations from the three-dose schedule were uncommon and across five clinical trials only $0.2 \%$ of subjects discontinued treatment due to the emergence of adverse events. ${ }^{9,21}$

\section{PHARMACOECONOMICS}

The introduction of HPV vaccines has the potential to considerably reduce the burden of HPV-related diseases and the substantial healthcare costs associated with them. ${ }^{18}$ Although the introduction of Gardasil will not preclude the need for routine cervical screening in the future, we can certainly expect the frequency of cervical abnormalities to diminish and the intervals between screening visits to increase, thereby reducing the cost and inconvenience of screening and any follow-up. ${ }^{18,23}$ Furthermore, costs associated with managing cases of clinical disease will also fall. ${ }^{23,24}$ By protecting against persistent infection by HPV types responsible for low-grade cervical lesions and other genital lesions, the high costs of managing genital warts and other lesions can also be expected to fall.

Pharmacoeconomic analyses have shown HPV vaccination to be cost-effective when used in conjunction with existing cervical cancer screening programmes. ${ }^{25}$ Cost-effectiveness is highly sensitive to the age at which vaccination is initiated, the duration of protection afforded by the vaccine, the direct costs of the vaccine and the intervals between routine cervical smears. A US analysis has shown that vaccinating girls from the age of 12 years and initiating cervical screening every 3 years from the age of 25 would reduce the overall lifetime risk of cervical cancer by $94 \%$ compared with no intervention. ${ }^{26}$ The cost-effectiveness of this approach was in line with many other currently accepted therapeutic interventions. ${ }^{27}$

Administering HPV vaccination prior to onset of sexual activity will yield the greatest health benefits. ${ }^{18}$ This assertion has been acknowledged in recent recommendations from the Advisory Committee on Immunization Practices (ACIP) in the USA, which has called for universal HPV vaccination for all girls aged 11-12 years. ${ }^{6}$ The ACIP has also suggested that vaccination could be started from the age of 9 and that a catch-up programme of immunisation in unvaccinated females aged 13-26 years will yield even greater benefits to public health. However, the introduction of

Table 2. Vaccine-related adverse events amongst vaccine recipients in the Gardasil clinical trial programme. ${ }^{9}$ Adverse events shown were reported with a frequency of $\geq 1 \%$ and at a greater frequency compared with the placebo cohort.

\begin{tabular}{ll}
\hline Frequency & Adverse event \\
\hline \multirow{2}{*}{ Very common $(\geq 1 / 10)$} & General disorders and injection site reactions \\
& Pyrexia \\
Common $(\geq 1 / 100$ but $<1 / 10)$ & Erythema, pain, swelling \\
\hline & Bleeding, pruritus \\
Rare $(\geq 1 / 10,000$ but $<1 / 1,000)$ & Skin and subcutaneous tissue disorders \\
\hline & Urticaria \\
Very rare $(<1 / 10,000)$ & Respiratory, thoracic and mediastinal disorders \\
\hline
\end{tabular}


HPV vaccines into the vaccine schedules of other countries has been delayed because of an ongoing debate regarding the appropriate age of vaccination, the fact that HPV infection indicates sexual activity and not necessarily sexual promiscuity and other issues surrounding the morality of vaccinating sexually naïve individuals.

\section{KEY POINTS}

- Gardasil has high efficacy in preventing persistent infection by HPV 6, 11, 16 and 18 and HPV-related CIN for up to 5 years.

- Gardasil was 100\% effective in preventing HPV 16/18-related CIN2/3, AIS and cervical cancer amongst women naïve to these HPV types at baseline.

- Gardasil also protects against low-grade cervical dysplasia and has high protective value against external genital lesions including VIN, ValN and anogenital warts.

- Gardasil is effective against the remaining virus types in women who are positive to one or more HPV vaccine types at enrolment.

- The vaccine-induced immune response is more robust than that associated with a natural HPV infection, and persists for at least 5 years.

- Gardasil is immunogenic in children and younger adolescents. Immune responses in this group are numerically greater than those seen in older adolescents and younger women, indicating the vaccine is also effective in this younger population.

\section{REFERENCES}

1. Bryan JT. Developing an HPV vaccine to prevent cervical cancer and genital warts. Vaccine 2007.

2. Parkin DM, Bray F. Chapter 2: The burden of HPV-related cancers. Vaccine 2006; 24 Suppl 3: S11-25.

3. Hymel PA. Decreasing risk: impact of HPV vaccination on outcomes. Am J Manag Care 2006; 12: S473-83.

4. Schmiedeskamp MR, Kockler DR. Human papillomavirus vaccines. Ann Pharmacother 2006; 40: 1344-52; quiz 1494-5.

5. Walboomers JM, Jacobs MV, Manos MM et al. Human papillomavirus is a necessary cause of invasive cervical cancer worldwide. 7 Pathol 1999; 189: 12-9.

6. Centers for Disease Control and Prevention (CDC). Advisory Committee on Immunization Practices (ACIP). ACIP provisional recommendations for the use of quadrivalent HPV vaccine. 15 August 2006. www.cdc.gov/nip/recs/provisional_recs/hpv.pdf.

7. Villa LL. Prophylactic HPV vaccines: reducing the burden of HPV-related diseases. Vaccine 2006; 24 Suppl 1: S23-8.

8. Shi L, Sings HL, Bryan JT et al. GARDASIL: prophylactic human papillomavirus vaccine development-from bench top to bed-side. Clin Pharmacol Ther 2007; 81: 259-64.

9. Ault K. Prophylactic use of quadrivalent human papillomavirus (types $6,11,16,18) \mathrm{L} 1$ virus-like particle vaccine reduces cervical intraepithelial neoplasia $2 / 3$ and adenocarcinoma in situ risk. Eur 7 Cancer Suppl 2005; 3: 11(PS10).
10. Villa LL, Costa RL, Petta CA et al. High sustained efficacy of a prophylactic quadrivalent human papillomavirus types 6/11/16/18 L1 virus-like particle vaccine through 5 years of follow-up. Br 7 Cancer 2006; 95: 1459-66.

11. Villa LL, Costa RL, Petta CA et al. Prophylactic quadrivalent human papillomavirus (types $6,11,16$, and 18) L1 virus-like particle vaccine in young women: a randomised double-blind placebo-controlled multicentre phase II efficacy trial. Lancet Oncol 2005; 6: 271-8.

12. Villa LL, Ault KA, Giuliano AR et al. Immunologic responses following administration of a vaccine targeting human papillomavirus Types 6, 11, 16, and 18. Vaccine 2006; 24: 5571-83.

13. Sattler C. Efficacy of a prophylactic quadrivalent human papillomavirus (types $6,11,16,18$ ) L1 virus-like particle vaccine for prevention of cervical dysplasia and external genital lesions. Abstract LB2-25. Presented at the 45th Interscience Conference on Antimicrobial Agents and Chemotherapy (ICAAC). 16-19 December 2005, Washington DC, USA.

14. Skjeldestad FE. Prophylactic quadrivalent human papillomavirus (types 6, 11, 16, 18) L1 virus-like particle vaccine (Gardasil) reduces cervical intraepithelial neoplasia 2/3 risk. Abstract LB-8. Presented at the 43rd Annual Meeting of the Infectious Diseases Society of America (IDSA). 6-9 October 2005, San Francisco, USA.

15. Stanley M. Prophylactic HPV Vaccines. 7 Clin Pathol 2007; 60: 961-965. 
16. Koutsky LA, Ault KA, Wheeler CM et al. A controlled trial of a human papillomavirus type 16 vaccine. $\mathcal{N}$ Engl 7 Med 2002; 347: 1645-51.

17. Mao C, Koutsky LA, Ault KA et al. Efficacy of human papillomavirus-16 vaccine to prevent cervical intraepithelial neoplasia: a randomized controlled trial. Obstet Gynecol 2006; 107: 18-27.

18. Soper D. Reducing the Health Burden of HPV Infection Through Vaccination. Infect Dis Obstet Gynecol 2006;14:83084.

19. Ferris D. Efficacy of a quadrivalent HPV (types $6 / 11 / 16 / 18) \mathrm{L} 1$ virus-like particle vaccine in women with virologic evidence of HPV infection: a combined analysis.

20. Leggatt GR, Frazer IH. HPV vaccines: the beginning of the end for cervical cancer. Curr Opin Immunol 2007; 19: 232-8.

21. May J. HPV vaccination - a paradigm shift in public health. Aust Fam Physician 2007; 36: 106-11.

22. Block SL, Nolan T, Sattler C, et al. Comparison of the immunogenicity and reactogenicity of a prophylactic quadrivalent human papillomavirus (types 6, 11, 16, and 18) L1 virus-like particle vaccine in male and female adolescents and young adult women. Pediatrics 2006; 118: 2135-45.
23. Brown RE, Breugelmans JG, Theodoratou D, Benard S. Costs of detection and treatment of cervical cancer, cervical dysplasia and genital warts in the UK. Curr Med Res Opin 2006; 22: 663-70.

24. Lowndes CM, Gill ON. Cervical cancer, human papillomavirus, and vaccination. BMF 2005; 331: 915-6.

25. Kulasingam SL, Myers ER. Potential health and economic impact of adding a human papillomavirus vaccine to screening programs. FAMA 2003; 290: 781-9.

26. Goldie SJ, Kohli M, Grima D et al. Projected clinical benefits and cost-effectiveness of a human papillomavirus 16/18 vaccine. $\mathcal{F}$ Natl Cancer Inst 2004; 96: 604-15.

27. Siddiqui MA, Perry CM. Human papillomavirus quadrivalent (types 6, 11, 16, 18) recombinant vaccine (Gardasil). Drugs 2006; 66: 1263-71; discussion 1272-3.

Correspondence:

Stephen I'Anson, Just Medical Media Ltd, 11 Redgrove Park, Cheltenham, Gloucestershire, GL51 6QY, UK.

Email: publisher@justmedicalmedia.com. 